\title{
Immunogenicity analysis of genetically conserved segments in Plasmodium ovale merozoite surface protein- 8
}

\author{
Xinxin Zhang ${ }^{1 \dagger}$, Ruilin Chu ${ }^{1 \dagger}$, Sui Xu², Haitian Fu' ${ }^{1}$, Jianxia Tang ${ }^{2}$, Limei Chen ${ }^{1}$, Xiaodan Shi ${ }^{1}$, Jing Chen², \\ Yuhong Li ${ }^{1}$, Guoding Zhu' ${ }^{2}$, Eun-Taek Han ${ }^{3}$, Yinghua Xuan ${ }^{1 *}$, Jun Cao ${ }^{1,2^{*}}$ and Yang Cheng ${ }^{1 *}$
}

\begin{abstract}
Background: Plasmodium ovale is widely distributed across tropical regions and has two closely related but distinct species, namely P. ovale curtisi and P. ovale wallikeri. Combining genetic characterization with the immunogenicity of merozoite surface protein-8 (MSP-8) supports considering MSP-8 as a candidate target for blood-stage vaccines against malaria. However, no previous studies have focused on characterizing the genetic diversity and immunogenicity of PoMSP-8.

Methods: Blood samples were collected from 42 patients infected with P. ovale. The patients were migrant workers returning to the Jiangsu Province from Africa; genomic DNA was extracted from their blood samples for sequencing and protein expression. The recombinant PoMSP-8 (rPoMSP-8) proteins were expressed and purified to assess their immune responses in BALB/c mice.

Results: The sequences of the P. ovale curtisi and P. ovale wallikeri msp8 genes were completely conserved in each isolate. The rPoMSP-8 proteins were successfully expressed and purified as $\sim 70 \mathrm{kDa}$ proteins. Antibodies raised against rPoMSP-8 in mice showed appropriate immunoreactivity, as evidenced by immunoblotting. These specific antibodies were detected at day 7 post-immunization, and their levels increased throughout the whole immunization period. rPoMSP-8-raised antibodies had high endpoint titers $(1: 5,120,000)$ and high avidity (PocMSP-8: 94.84\%, PowMSP-8: 92.69\%). Cross-reactivity between rPocMSP-8 and rPowMSP-8 was observed with each anti-PoMSP8-specific antibody.

Conclusions: Remarkable conservation and high immunogenicity was observed in both rPoMSP-8s. Intriguingly, cross-reaction between rPocMSP-8 and rPowMSP-8 was detected, suggesting that a single PoMSP8-based construction might be applicable for both species.
\end{abstract}

Keywords: Plasmodium ovale, MSP-8, Immunogenicity, Cross-reactivity, Conservation

\section{Background}

Malaria remains a disease of global health importance [1]. In 2017, an estimated 219 million cases of malaria and 435,000 malaria-caused deaths were reported [2]. One causative agent of human malaria, Plasmodium

\footnotetext{
*Correspondence: yhxuan@jiangnan.edu.cn; caojuncn@hotmail.com; woerseng@126.com

${ }^{\dagger}$ Xinxin Zhang and Ruilin Chu contributed equally to this work

${ }^{1}$ Laboratory of Pathogen Infection and Immunity, Department of Public Health and Preventive Medicine, Wuxi School of Medicine, Jiangnan

University, Wuxi, Jiangsu, People's Republic of China

Full list of author information is available at the end of the article
}

ovale, was first described by Stevens [3]. Ovale malaria in humans is caused by two closely related but distinct subspecies of the malarial parasite, $P$. ovale curtisi (the classic type) and $P$. ovale wallikeri (the variant type) [4]. Little attention has been paid to ovale malaria because it often presents with low parasitemia and mixed infection with other Plasmodium species, with mild clinical symptoms that are easily treated with the conventional antimalarial drug chloroquine [5-9].

Jiangsu Province, located in eastern China, was previously an unstable malaria-transmission area. After large-scale implementation of interventions such as mass 
drug administration, indoor residual spraying, and longlasting insecticide nets, malaria has been effectively controlled in the Jiangsu Province [10], and no report of local malaria infection has been issued since 2012 [11]. With the continuous expansion and deepening of the ChinaAfrica cooperation, large numbers of Chinese people travel to malaria-endemic countries for trade, tourism, labor and other purposes [12]. Between 2014 and 2016, 1068 imported cases of malaria were reported in the Jiangsu Province, and approximately 170 cases of malaria in China imported from Africa were caused by P. ovale [13].

Parasite antigenic diversity has become a major obstacle hindering the development of effective malaria vaccines [14]. Erythrocyte invasion is a critical point in the life-cycle of malaria parasites and it is at this point that merozoites can be targeted by antibodies and eliminated by immune cells [15]. This type of vaccine generally incorporates antigenic proteins that are highly expressed on, or associated with, the surface of merozoites, such as the merozoite surface proteins (MSPs) [14]. MSPs comprise some of the best characterized proteins in $P$. falciparum and some of them are considered promising vaccine candidates due to their accessibility to interaction with host immune system molecules [15]. MSP-8 is a glycosylphosphatidylinositol (GPI)-anchored protein produced by blood-stage malaria parasites. MSP-8 contains a signal sequence at the $\mathrm{N}$-terminus and two epidermal growth factor (EGF)-like domains at the C-terminus with significant homology to those of other Plasmodium species. Serum from patients infected with $P$. falciparum could react against several PfMSP-8 fragments [16]. Likewise, $P$. vivax MSP-8 could induce humoral and cellular immune responses during $P$. vivax infection [17]. These data demonstrated that both PfMSP-8 and PvMSP- 8 were immunogenic during natural infection. Immunization with $P$. yoelii MSP-8 (PyMSP-8) induced a level of protection in mice to lethal $P$. yoelii challenge [18]. Thus, PyMSP-8 was a target of protective immune responses. MSP- 8 proteins are highly conserved across all Plasmodium sp. [19]. The conservation of MSP- 8 proteins support their consideration as part of malaria vaccine formulations [20].

Therefore, MSP- 8 from various malarial parasite species have been considered good vaccine candidates. However, insufficient evidence exists regarding the diversity and immunogenicity of P. ovale MSP-8 (PoMSP-8). In this study, $m s p 8$ sequences were analyzed from both $P$. ovale sp. obtained from infected migrant workers returning to China from Africa. Sequence analysis of the pomsp 8 genes showed complete conservation. The recombinant PoMSP-8 (rPoMSP-8) proteins were produced, and high immunogenicity was detected in immunized mice.

\section{Methods}

\section{Study areas and sample collection}

$P$. ovale curtisi and $P$. ovale wallikeri samples were obtained at local hospitals in the Jiangsu Province in China between 2012 and 2016 from febrile patients who had recently returned from working in tropical regions of sub-Saharan Africa, endemic for malaria [21]. Identification of the isolates was confirmed by polymerase chain reaction (PCR) analysis and Plasmodium species were distinguished by real-time TaqMan PCR [11]. A total of $42 P$. ovale-infected blood samples were randomly selected and analyzed. As a result, we identified 24 P. ovale curtisi samples and 18 P. ovale wallikeri samples. Full details of the isolates were provided (Additional file 1: Table S1).

\section{Cloning and sequencing}

The full-length sequences of pocmsp 8 and powmsp 8 were amplified by PCR using the primers pocmsp8Forward (5'-ATG GTT ATG ATT ATG AAA AAG AA- $3^{\prime}$ ) and pocmsp8-Reverse (5'-CTA TAA TAA ATA TAC ACA TAA CAT C-3'), and powmsp8-Forward (5'-ATG GTT ATG ATT ATG AAA AAG AA-3') and powmsp8-Reverse (5'-CTA TAA TAA ATA TAC ACA TAA CAT C-3'), respectively. The pocmsp 8 (PlasmoDB, PocGH01_10040100) and powmsp 8 (GenBank accession number, SBT74417.1) sequences from the Plasmodium Genomics Resource database and the National Center for Biotechnology Information GenBank database were used as reference gene sequences. The reactions were performed in a $20 \mu \mathrm{l}$ reaction volume, including $1 \mu \mathrm{l}$ of genomic DNA, $7.4 \mu \mathrm{l}$ of double-distilled water, $0.8 \mu \mathrm{l}$ of each primer, 0.5 units of DNA polymerase, $2 \mathrm{mM}$ deoxynucleoside triphosphate and $10 \mu$ lof premix $\left(2 \times\right.$ Phanta $^{\circledR}$ Max Master Mix, Vazyme, Nanjing, China). PCR amplification was performed in a Mastercycler (Eppendorf, Hamburg, Germany) with the following program: denaturation at $95{ }^{\circ} \mathrm{C}$ for $3 \mathrm{~min} ; 35$ cycles of $95^{\circ} \mathrm{C}$ for $15 \mathrm{~s}$, $45{ }^{\circ} \mathrm{C}$ for $15 \mathrm{~s}$ and $72{ }^{\circ} \mathrm{C}$ for $30 \mathrm{~s}$; and a final extension at $72{ }^{\circ} \mathrm{C}$ for $5 \mathrm{~min}$. The amplified products were analyzed by $1 \%$ agarose gel electrophoresis and visualized under an ultraviolet transilluminator (ChemiDoc MP, Bio-Rad, Hercules, CA, USA). The PCR product sizes were estimated based on the mobilities relative to a standard DNA marker (Transgen Biotech, Beijing, China). PCR products were cloned into the pUC57 vector and sequenced using universal primers (M13F: 5'-TGT AAA ACG ACG GCC AGT-3'; M13R: 5'-CAG GAA ACA GCT ATG AC-3'), which was performed by GENEWIZ (Suzhou, China) on an ABI 3730xl DNA Analyzer (Thermo Fisher Scientific, Waltham, MA, USA). 


\section{Sequence alignment and data analysis}

The primary structure of the PoMSP- 8 protein was predicted with a bioinformatics tool (http://smart.emblheidelberg.de/). To evaluate the diversity of both $P$. ovale sp., the pocmsp 8 and powmsp 8 sequences were used as templates and aligned using GeneDoc v.2.7.0.

\section{Recombinant protein expression and purification}

The pomsp 8 gene were subcloned into the pET32a expression vector (YouLong Biotech, Shanghai, China). This vector adds thioredoxin and six-histidine tags at both the $\mathrm{N}$ - and $\mathrm{C}$-terminal ends, enabling easier purification and immunodetection using monoclonal antibodies against the six-histidine tag. Next, rosetta-gami bacteria (Transgen Biotech) were transformed with the recombinant plasmids. This strain contains a plasmid that encodes additional copies of rare Escherichia coli (E. coli) tRNAs for enhanced expression of foreign proteins. Proteins were purified by YouLong Biotech. Protein expression was verified by $8 \%$ sodium dodecyl sulfatepolyacrylamide gel electrophoresis (SDS-PAGE) with Coomassie blue staining or western blot analysis.

\section{Mice immunizations}

Female BALB/c mice, 6-8 weeks of age, were used for immunizations (rPoMSP-8-immunized group and the negative-control group, $n=4$ per group). Purified rPoMSP-8, $30 \mu \mathrm{g}$, was injected into each mouse. Complete Freund's adjuvant (CFA; Sigma, San Francisco, CA, USA) was used for the primary booster, and incomplete Freund's adjuvant (IFA; Sigma, San Francisco, CA, USA) was used for the subsequent boosters. Boosters were administered on days 21 and 42 post-immunization. All injections were given intraperitoneally. The serum of each mouse was collected at days $0,7,14,28,35$ and 49 post-immunization. Mice in the negative-control group were injected with a mixture of PBS and adjuvant.

For western immunoblot analysis, rPoMSP-8 antigens were electrophoretically transferred from SDS-PAGE gels to $0.2 \mu \mathrm{m}$ polyvinylidene fluoride membranes (Millipore, Darmstadt, Germany) and probed with serum pooled from mice immunized with recombinant proteins acquired at day 49 and diluted 1:1000. Immunoreactivity was detected using a polyclonal goat anti-mouse antibody as a secondary antibody (Southern Biotech, Tuscaloosa, AL, USA).

\section{Antibody responses}

Antibody responses to PoMSP-8 at 0, 7, 14, 28, 35 and 49 days after primary immunization were measured by performing enzyme-linked immunosorbent assays
(ELISAs). Briefly, $50 \mathrm{ng}$ of rPoMSP-8 antigens were coated overnight at $4{ }^{\circ} \mathrm{C}$ in 96-well ELISA plates (Corning, Corning, NY, USA). After blocking with $1 \times$ PBS containing $1 \%$ bovine serum albumin (BSA; Beyotime Biotechnology, Beijing, China), the serum samples were diluted 1:20,000 in $1 \times$ PBS containing Tween 20 (PBS$\mathrm{T}$ ) and $0.5 \%$ BSA, and incubated in duplicate wells for $90 \mathrm{~min}$. Horseradish peroxidase-conjugated goat antimouse IgG antibodies (Southern Biotech) were diluted 1:5000 and used to detect the IgG antibodies bound to PoMSP-8. The reaction was developed by adding the 3 , $3^{\prime}, 5,5^{\prime}$-tetramethylbenzidine (Invitrogen, Waltham, MA, USA) as a substrate and then stopped by adding $2 \mathrm{M}$ $\mathrm{H}_{2} \mathrm{SO}_{4}$; the absorbance was then measured at $450 \mathrm{~nm}$. To evaluate the titration of specific anti-PoMSP- 8 antibodies, sera from mice in each group were pooled and used in serial dilutions $(1: 10,000$ to $1: 5,120,000)$ in ELISA.

The avidity of anti-rPoMSP-8 IgG antibodies was estimated as described previously [22]. Briefly, ELISA testing was performed as described above, except that the test was performed in duplicate plates. After diluting the sera $(1: 20,000)$ and incubating for $90 \mathrm{~min}$, one of the plates was washed three times with PBS-T, and the other plate was incubated with the same sera and washed three times with dissociation buffer, comprised of PBS-T with $6 \mathrm{M}$ urea. Then, the plates were washed once with PBS-T buffer. Incubation with a secondary antibody, the wash steps, and developing the enzyme reaction were performed as described above for the ELISAs. The avidity index (AI) for each sample was calculated as follows:

$$
\begin{aligned}
& \mathrm{AI}=\left(\mathrm{OD}_{450} \text { of a sample treated with } 6 \mathrm{M}\right. \text { urea/ } \\
& \left.\quad \mathrm{OD}_{450} \text { of a sample not treated with } 6 \mathrm{M} \text { urea }\right) \times 100 .
\end{aligned}
$$

\section{Statistical analysis}

Statistical analysis and graphing were done using GraphPad Prism software, v.5.0 (GraphPad Software Inc., San Diego, CA, USA). To analyze cross-reaction and antibody responses, the data were analyzed by SPSS v.16.0. Student's t-test was used to analyzed data emerging on normal distribution, with probability $(P)$ values $>0.05$ considered to reflect not statistically significant differences. Phylogenetic trees for MSP-8 were constructed using the neighbor-joining method based on the nucleotide sequences. Phylogenetic relationships based on the msp 8 gene were estimated using the neighbor-joining (NJ) method in MEGA v.7.0, the general time reversible (GTR) nucleotide model with a gamma $(\gamma)$-distribution model of among-site rate variation and a proportion of invariable sites (i.e. the GTR $+\gamma+\mathrm{I}$ substitution model) determined. The evolutionary relationships of the aligned sequences were determined using MEGA v.7.0. 


\section{Results}

\section{Description and gene analysis of PoMSP-8}

The single-exon $P$. ovale curtisi and $P$. ovale wallikeri msp 8 genes were both found to be 1446 base pairs long and to begin with a predicted 28-amino acid (aa) signal peptide sequence (aa 1-28). Some other specific regions were identified in the predicted primary structure of the pomsp8, such as a coiled-coil domain (aa 100-121), two epidermal growth factor (EGF)-like domains (aa 376-415 and 421-458), a transmembrane region (aa 461-480) and a GPI-anchor (aa 457-481) (Fig. 1a). To verify this putative gene structure for pomsp8, we prepared genomic DNA from patients infected with $P$. ovale and used it as the PCR template. Pomsp 8 genes were successfully amplified by PCR in all isolates, generating single PCR products of the expected size $(\sim 1.5 \mathrm{~kb})$. Direct sequencing of these purified PCR fragments showed no superimposed signal on the electropherograms for pomsp8 (Fig. 1b). Overall, the full length alignments for pomsp8 showed that no amino acid mutations occurred among the 24 pocmsp 8 samples and 18 powmsp 8 samples originating from 15 countries in sub-Saharan Africa (Additional file 2: Figure S1). These findings suggested that the pomsp 8 genes were completely conserved in each isolate.

\section{Phylogenetic analysis}

Based on the high level of genetic conservation presented above, phylogenetic analysis was performed using $m s p 8$ gene sequences from human, non-human primate, murine and avian malarial species, using the neighbor-joining method. This analysis showed that pocmsp 8 and powmsp 8 occupied a distinct bifurcating branch with 98 and 95\% bootstrap support, respectively (Fig. 2). The $m s p 8$ gene ID number of other Plasmodium species included in the analyses are provided in Additional file 3: Table S2.
Expression and purification of recombinant PoMSP-8

Truncated PoMSP-8 $(\Delta \mathrm{SP})$ proteins are predicted to consist of 430 aa residues with a molecular weight of $52 \mathrm{kDa}$. Both recombinant soluble forms of PoMSP-8 were successfully expressed using $E$. coli expression system and purified using an Ni-Sepharose column. The purity of the recombinant PoMSP-8 proteins were assessed. The pET32a adds thioredoxin and six-histidine tags at both the $\mathrm{N}$ - and $\mathrm{C}$-terminal ends, so the proteins migrated with a molecular weight of $\sim 70 \mathrm{kDa}$, based on SDS-PAGE analysis (Fig. 3a). Moreover, the corresponding immunoblots probed with an anti-His tag antibody confirmed that rPoMSP-8 was expressed (Fig. 3b). The confirmed proteins were used to immunize mice.

\section{Mice antibodies against PoMSP- 8 recognized the recombinant proteins}

To determine whether mice anti-rPoMSP8 antibodies could recognize the rPoMSP-8s, we developed an immunoblot for a specific $\sim 70 \mathrm{kDa}$ band that should be detected for both PoMSP-8s. The antibody responses of immunized mouse sera against PoMSP-8 were potent, suggesting that PoMSP-8 is highly immunogenic in mice. As a negative control, no reactivity was observed in sera from mice before immunization (Fig. 4a). To identify cross-reaction between PocMSP- 8 and PowMSP-8, the antibodies raised against PowMSP- 8 were reacted with rPocMSP-8, and mouse antiserum raised against PocMSP-8 were cross-reacted with rPowMSP-8 (Fig. 4b, c, $P>0.05)$. Then, cross-reactivity was verified using anti-rPoMSP-8 immune mouse serum with rPoMSP$8 \mathrm{~s}$ (Fig. 4d). These results showed that rPoMSP-8 could induce immune response in mice and the mice antirPoMSP8 antibodies could recognize the rPoMSP-8s.

\section{Immune responses against rPoMSP-8 in mice}

To begin characterizing immune responses generated by immunization with PoMSP-8, groups of $4 \mathrm{BALB} / \mathrm{c}$ mice $\mathbf{a}$

PocMSP-8 and PowMSP-8, 481 aa. (54kDa)

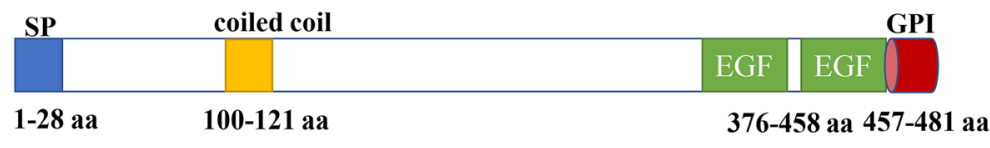

Protein expression: 29-481 aa (52 kDa)

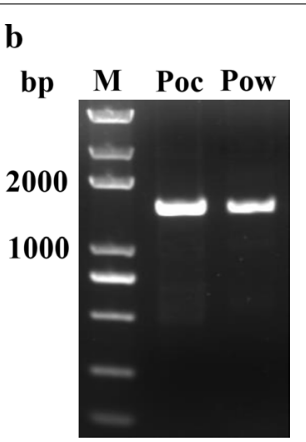

Fig. 1 Schematic representation of the gene structure and amino acid sequence alignment of $P$. ovale MSP-8 C-terminal fragment. a Diagram of the gene structure of pomsp-8. Both pomsp-8 genes encode a signal peptide (blue), a coiled-coil domain (yellow), two EGF-like domains (green) and a GPI-anchor (red). $\mathbf{b}$ The full-length msp-8 genes of 42 P. ovale isolates were amplified by PCR 


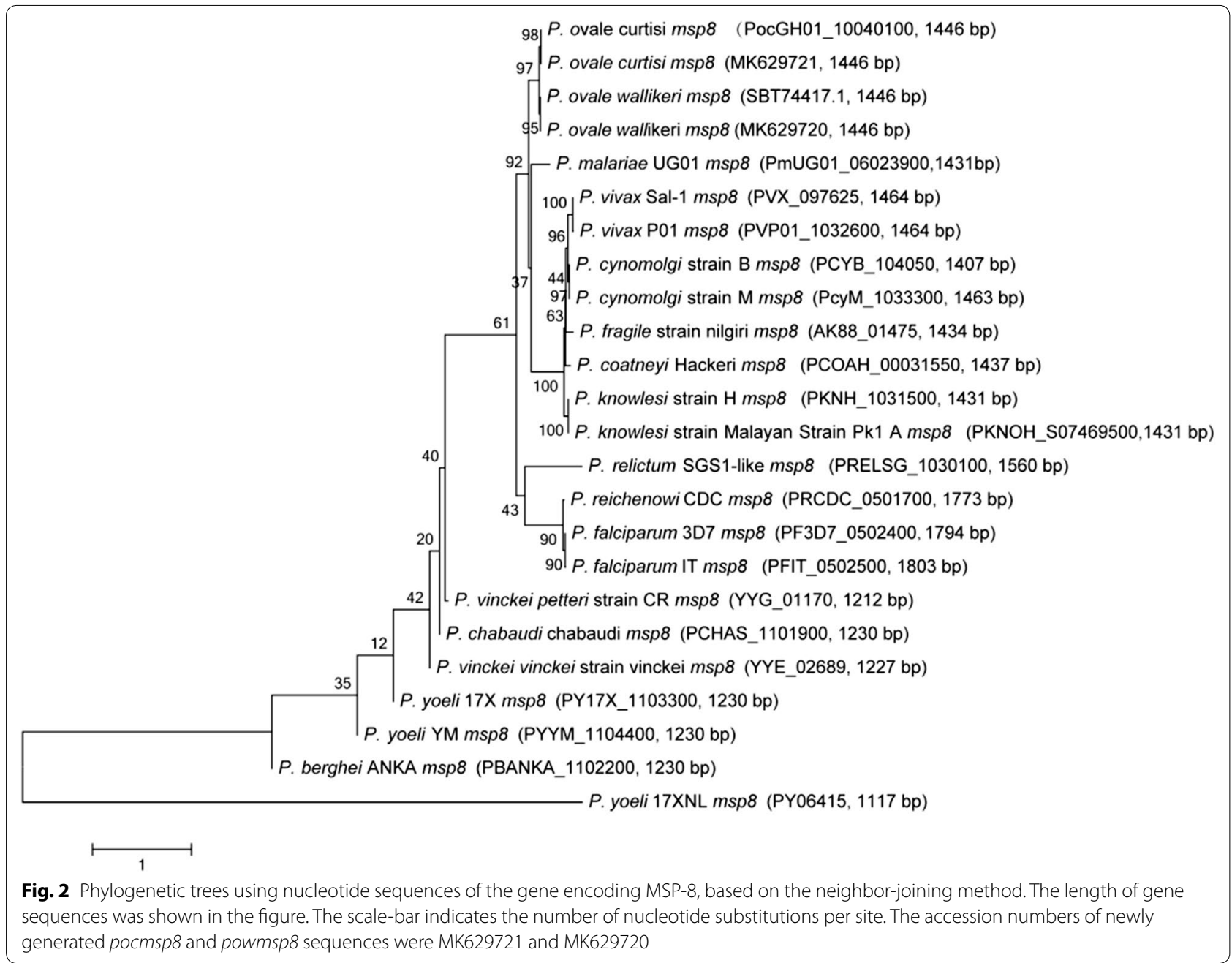

were immunized with rPocMSP-8, rPowMSP-8, or PBS as a control, and the resultant antibody responses were measured by ELISA using the same recombinant proteins as the solid-phase coating antigen. The results provided in Fig. 5 show that both recombinants were immunogenic. ELISA results showed that antibodies against PoMSP-8 were detected one week after the primary booster. A high response was detected at day 14 postimmunization and continued to rise until day 28 postimmunization (Fig. 5a).

The mean serum antibody titers were evaluated 49 days after first immunization by ELISA. Similarly, PocMSP-8 and PowMSP-8 were linearly detected in ELISAs, and the PocMSP-8 and PowMSP-8 antigens could induce comparable IgG end-point titers (1:5,120,000; Fig. 5b).

In this study, high-avidity IgG antibodies were induced in all mouse groups immunized with adjuvant formulations. A higher IgG avidity response was detected in mice immunized with PocMSP-8 (mean: 94.84\%) than was detected in mice immunized with PowMSP-8 (mean:
94.89\%), but this difference was not statistically significant $(P>0.05)$.

\section{Discussion}

Investigations into the extent of sequence variation amongst malaria vaccine candidates are undoubtedly important as the basis of effective malaria vaccine development. Here, we analyzed 42 clinical isolates from imported cases from Africa. The full length of pomsp8 sequences (24 pocmsp 8 isolates and 18 powmsp 8 isolates) were completely conserved. The high degree of conservation observed among samples from 15 different African countries suggests that pomsp 8 may be useful in PCRbased diagnostic testing for $P$. ovale. This result is consistent with other reports in terms of MSP-8 sequence conservation in $P$. falciparum, $P$. vivax and $P$. yoelii $[19$, 20]. These findings suggest that the degree of conservation could be the result of maintained functions during red blood cell invasion and/or that these domains 


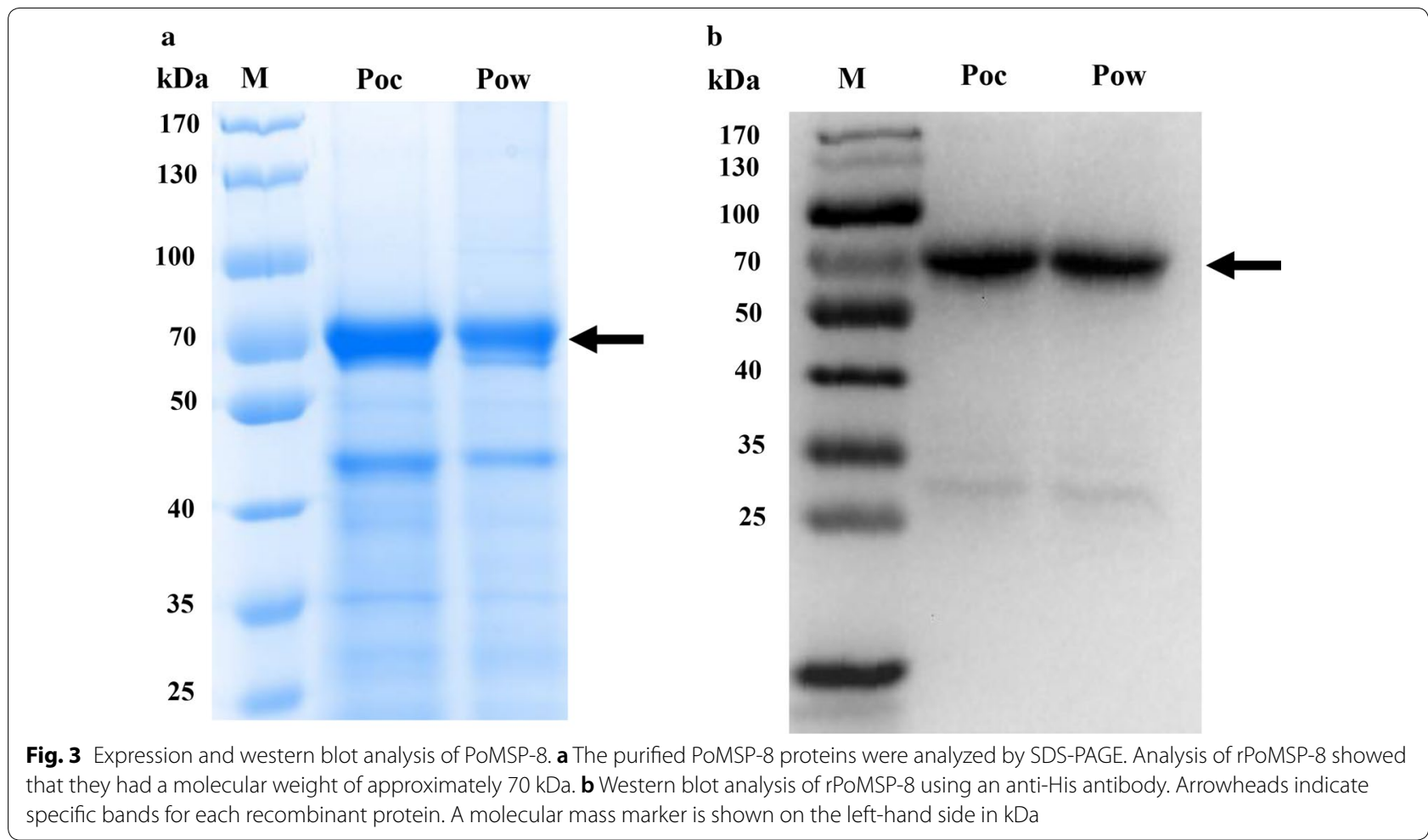

perform related or redundant roles in the parasite's lifecycle $[23,24]$.

The msp 8 sequences placed $P$. ovale curtisi and $P$. ovale wallikeri in a distinct bifurcating branch in the phylogenetic tree and the results support an ancient time of divergence for the malarial parasite lineage [25]. This phylogenetic arrangement clearly indicates that $P$. malariae forms an outgroup both to rodent-infective species and to a primate-infective clade that includes $P$. vivax [26]. Whereas we found no conclusive evidence of selection acting on the polymorphism of $P$. ovale, likely due to the limited sample size, our comparative analyses based on phylogenetic methods indicate that msp 8 genes diverged under strong negative (purifying) selection, indicating functional constrains. The evidence that these proteins were conserved due to the potential action of purifying selection during their evolutionary history provides additional support for their consideration in malaria vaccine formulations.

Polymorphisms and immunogenicity are both within the scope of consideration when screening for new preclinical vaccine candidates. It had been reported that conserved regions were less antigenic and immunogenic than polymorphic regions [27-29]. Interestingly, the high conservation seen in PoMSP-8 mice was associated with a high level of immunogenicity, as both antibody titers were 1:5,120,000. This fragment of MSP-8 was expressed on the merozoite surface through a GPI-anchor and is taken up into erythrocytes [30]. GPI-anchors were previously found to be potent agonists of the toll-like receptor, as were adjuvants that facilitate immune responses [31]. As a surface protein, MSP-8 was exposed to the host immune system.

Antibodies play essential roles in protecting against blood-stage malaria. In this study, sera from mice immunized showed positive reactivity with rPocMSP-8 and rPowMSP-8, which are recombinant proteins. The fact that PoMSP- 8 elicited humoral immune responses supports the conclusion that the conserved sequences contain B epitopes. Cross-reaction between PocMSP-8 and PowMSP- 8 was detected by both ELISA and western immunoblot analyses. These findings suggested that rPoMSP-8 shares similar and conserved antigenic determinants and that a complex network of cross-reactivity exists between $P$. ovale sp. The finding may explain the diversity between different species and enable measurements of species-specific efficacy in vaccine trials.

Production of high-avidity antibodies is essential for preventing severe disease during malaria infections. Our results indicated that both PocMSP- 8 and PowMSP-8 induced high avidity antibodies (PocMSP-8: 94.84\%, PowMSP-8: 92.69\%). High-quality antibodies represent an important factor involved in preventing and protecting against infections [32]. These findings suggest that 


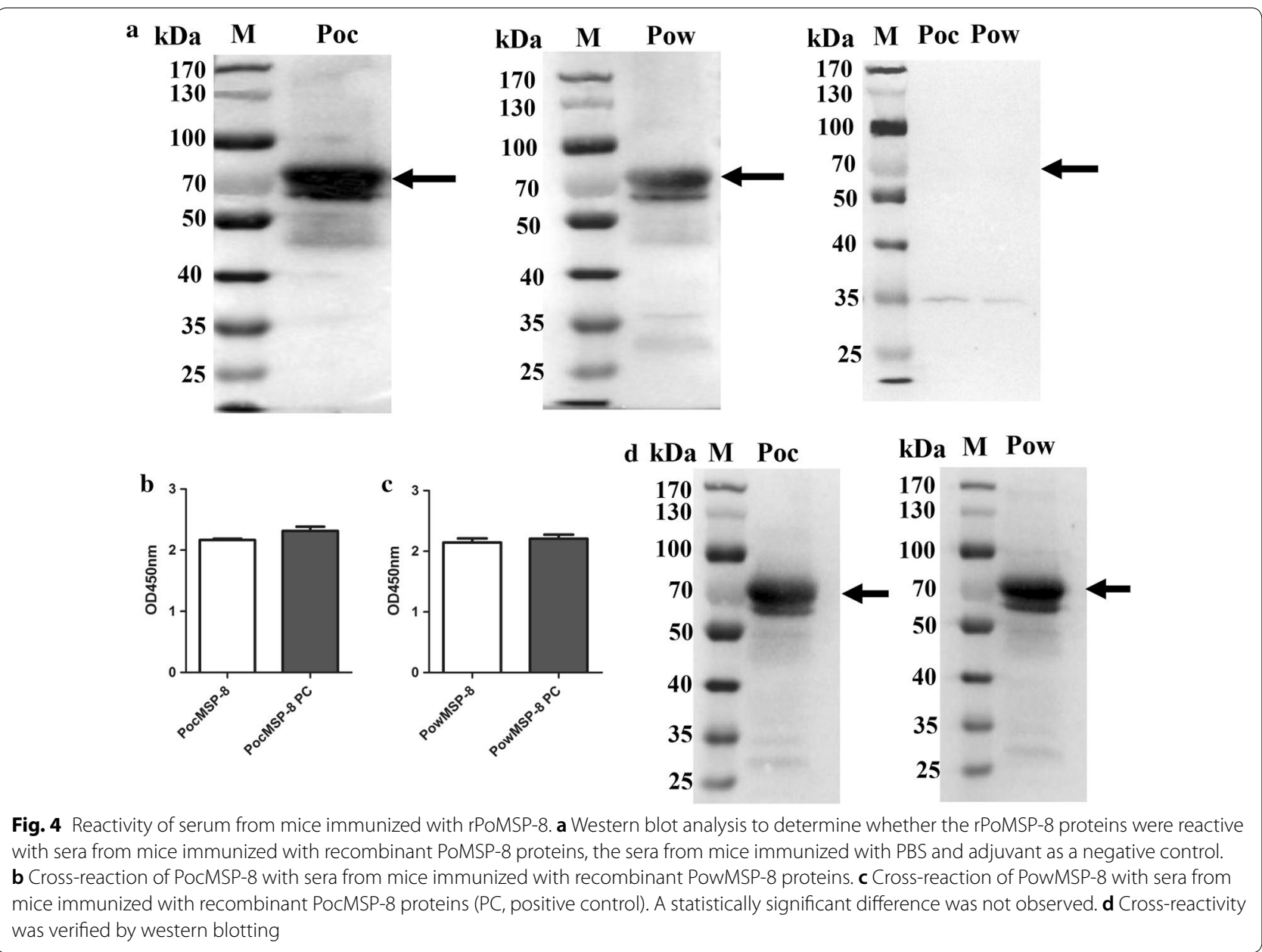
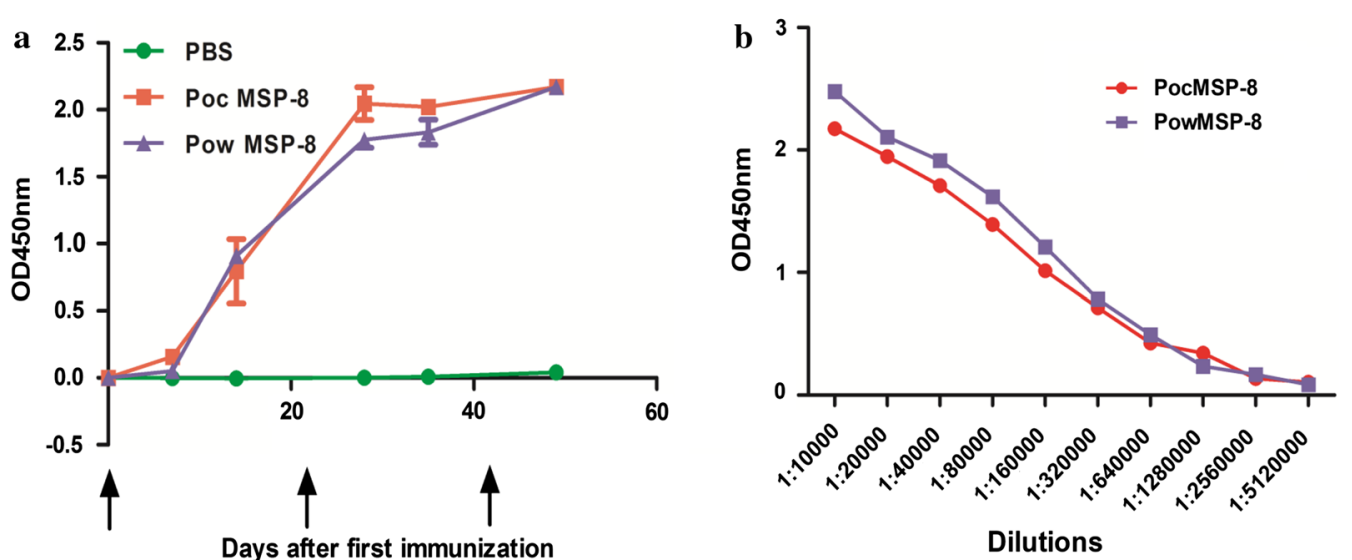

Fig. $5 \mathrm{Immune}$ responses in PoMSP-8-immunized mice. a lgG levels in PoMSP-8-immunized mice. IgG was detected at day 7 post-immunization, and the levels increased throughout the whole immunization period. $\mathbf{b}$ Data are presented as the geometric mean OD obtained at different concentrations, expressed as the reciprocal of the serum dilution obtained from mice immunized with PocMSP-8 or PowMSP-8 (dilutions from $1: 10,000$ to $1: 5,120,000)$. Numbers on the $X$-axis indicate the dilutions tested. Antigen specificity was confirmed using pre-immune serum samples as a control 
MSP-8 in mice is a valid model for understanding the immune response and may compensate for the difficulty of culturing this parasite in vitro. As such, it warrants further study.

\section{Conclusions}

The findings of this study demonstrate the remarkable conservation and high immunogenicity of both PoMSP8s. Cross-reaction between PocMSP-8 and PowMSP-8 was detected, suggesting that immune responses may be mediated by a repertoire of antibodies to common epitopes between both $P$. ovale subspecies. These data provide valuable reference information regarding the genetic diversity and immunogenicity of $P$. ovale curtisi and $P$. ovale wallikeri isolates imported from Africa to China. Therefore, PoMSP-8 may potentially serve as a candidate for malaria vaccine design, although further evaluation needs be carried out to validate its potential and limitations.

\section{Additional files}

Additional file 1: Table S1. Information on imported $P$. ovale curtisi and $P$. ovale wallikeri.

Additional file 2: Figure S1. Amino acid sequence alignment of $P$. ovale MSP-8 full length. a P. ovale curtisi. b P. ovale wallikeri.

Additional file 3: Table S2. The msp8 Gene ID number of other Plasmodium species.

\section{Abbreviations}

MSP-8: merozoite surface protein-8; rPoMSP-8: recombinant PoMSP-8; GPI: glycosylphosphatidylinositol; PCR: polymerase chain reaction; aa: amino acid EGF: epidermal growth factor; ELISA: enzyme-linked immunosorbent assays; Al: avidity index.

\section{Acknowledgements}

We thank all study participants, local health officials and doctors for their participation and support.

\section{Funding}

This study was funded by grants from the National Natural Science Foundation of China (81601787, 81871681), by the Natural Science Foundation of Jiangsu Province (BK20160192, BK20150001), by the Jiangsu Provincial Key Research and Development Program (BE2016631), by the Fundamental Research Funds for the Central Universities funded by the Ministry of Education of China (JUSRP51710A), by the Bill and Melinda Gates Foundation (OPP1 161962), by the national first-class discipline program of Food Science and Technology (JUFSTR20180101), and by the Jiangsu Provincial Project of Invigorating Health Care through Science, Technology and Education.

\section{Availability of data and materials}

The data supporting the conclusions of this article are included within the article and its additional files. The newly generated sequences were submitted in the GenBank database under the accession numbers MK629721 and MK629720.

\section{Authors' contributions}

$Y C$ and $Y H X$ conceived this study. XXZ designed the research protocol and wrote the manuscript. SX, JXT, JC and GDZ collected the samples. XXZ, RLC and LMC performed the acquisition of data and data analysis. XXZ, RLC, HTF and $\mathrm{YHX}$ conducted the laboratory work, data handling and analysis and reviewed the manuscript. HTF, ETH, XDS and YHL contributed to interpretation of the results and assisted in writing the manuscript. All authors read and approved the final manuscript.

\section{Ethics approval and consent to participate}

This study was approved by the Ethics Committee, Jiangsu Provincial Key Laboratory on Parasite and Vector Control Technology, Jiangsu Institute of Parasitic Diseases (JIPD) (IRB00004221), Wuxi, China. Informed consent was obtained from all of the participants, and the animal trial was approved by the Animal Ethics Committee, Jiangnan University (JN. no. 20180330b0480604).

\section{Consent for publication}

Not applicable.

\section{Competing interests}

The authors declare that they have no competing interests.

\section{Publisher's Note}

Springer Nature remains neutral with regard to jurisdictional claims in published maps and institutional affiliations.

\section{Author details}

${ }^{1}$ Laboratory of Pathogen Infection and Immunity, Department of Public Health and Preventive Medicine, Wuxi School of Medicine, Jiangnan University, Wuxi, Jiangsu, People's Republic of China. ${ }^{2}$ Key Laboratory of National Health and Family Planning Commission on Parasitic Disease Control and Prevention, Jiangsu Provincial Key Laboratory on Parasite and Vector Control Technology, Jiangsu Institute of Parasite Diseases, Wuxi, Jiangsu, People's Republic of China.

${ }^{3}$ Department of Medical Environmental Biology and Tropical Medicine, School of Medicine, Kangwon National University, Chuncheon, Gangwon-do, Republic of Korea.

Received: 4 November 2018 Accepted: 26 March 2019

Published online: 11 April 2019

\section{References}

1. Cowman AF, Healer J, Marapana D, Marsh K. Malaria: biology and disease. Cell. 2016;167:610-24.

2. WHO. World malaria report (2018). Geneva: World Health Organization; 2018.

3. Stephens JWW. A new malaria parasite of man. Ann Trop Med Parasitol. 1922;16:383-8.

4. Sutherland CJ, Tanomsing N, Nolder D, Oguike M, Jennison C, Pukrittayakamee $\mathrm{S}$. Two nonrecombining sympatric forms of the human malaria parasite Plasmodium ovale occur globally. J Infect Dis. 2010;201:1544-50.

5. Zhou R, Liu Y, Li S, Zhao Y, Huang F, Yang C, et al. Polymorphisms analysis of the Plasmodium ovale tryptophan-rich antigen gene (potra) from imported malaria cases in Henan Province. Malar J. 2018;17:127.

6. Roucher C, Rogier C, Sokhna C, Tall A, Trape JF. A 20-year longitudinal study of Plasmodium ovale and Plasmodium malariae prevalence and morbidity in a West African population. PLoS One. 2014;9:e87169.

7. Doderer-Lang C, Atchade PS, Meckert L, Haar E, Perrotey S, Filisetti D, et al. The ears of the African elephant: unexpected high seroprevalence of Plasmodium ovale and Plasmodium malariae in healthy populations in western Africa. Malar J. 2014;13:240.

8. Faye FBK, Spiegel A, Tall A, Sokhna C, Fontenille D, Rogier C, et al. Diagnostic criteria and risk factors for Plasmodium ovale malaria. J Infect Dis. 2002;186:690-5.

9. Oguike MC, Betson M, Burke M, Nolder D, Stothard JR, Kleinschmidt I, et al. Plasmodium ovale curtisi and Plasmodium ovale wallikeri circulate simultaneously in African communities. Int J Parasitol. 2011:41:677-83.

10. Hsiang MS, Hwang J, Tao AR, Liu Y, Bennett A, Shanks GD, et al. Mass drug administration for the control and elimination of Plasmodium 
vivax malaria: an ecological study from Jiangsu province, China. Malar J. 2013;12:383.

11. Cao Y, Wang W, Liu Y, Cotter C, Zhou H, Zhu G, et al. The increasing importance of Plasmodium ovale and Plasmodium malariae in a malaria elimination setting: an observational study of imported cases in Jiangsu Province, China, 2011-2014. Malar J. 2016;15:459.

12. Liu Y, Hsiang MS, Zhou H, Wang W, Cao Y, Gosling RD, et al. Malaria in overseas labourers returning to China: an analysis of imported malaria in Jiangsu Province, 2001-2011. Malar J. 2014;13:29.

13. Cao YY, Wang WM, Zhou HY, Zhu GD, Xu S, Gu YP, et al. Cases diagnosis of imported malaria in Jiangsu Province, 2014-2016. Chin J Epidemiol. 2018;39:218 (In Chinese).

14. Richie TL, Saul A. Progress and challenges for malaria vaccines. Nature. 2002:415:694-701.

15. Barry $A E$, Arnott $A$. Strategies for designing and monitoring malaria vaccines targeting diverse antigens. Front Immunol. 2014;5:359.

16. Black CG, WU T, Wang L, Hibbs AR, Coppel RL. Merozoite surface protein 8 of Plasmodium falciparum contains two epidermal growth factor-like domains. Mol Biochem Parasitol. 2001;114:217-26.

17. Cheng Y, Wang B, Changrob S, Han JH, Sattabongkot J, Ha KS, et al. Naturally acquired humoral and cellular immune responses to Plasmodium vivax merozoite surface protein 8 in patients with P. vivax infection. Malar J. 2017;16:211.

18. James M, Burns J, Belk CC, Dunn PD. A protective glycosylphosphatidylinositol-anchored membrane protein of Plasmodium yoelii trophozoites and merozoites contains two epidermal growth factor-like domains. Infect Immun. 2000;68:6189-95.

19. Alaro JR, Angov E, Lopez AM, Zhou H, Long CA, Burns JM Jr. Evaluation of the immunogenicity and vaccine potential of recombinant Plasmodium falciparum merozoite surface protein 8. Infect Immun. 2012;80:2473-84.

20. Pacheco MA, Elango AP, Rahman AA, Fisher D, Collins WE, Barnwell $\mathrm{JW}$, et al. Evidence of purifying selection on merozoite surface protein 8 (MSP8) and 10 (MSP10) in Plasmodium spp. Infect Genet Evol. 2012;12:978.

21. Chu R, Zhang X, Xu S, Chen L, Tang J, Li Y, et al. Limited genetic diversity of N-terminal of merozoite surface protein-1 (MSP-1) in Plasmodium ovale curtisi and P. ovale wallikeri imported from Africa to China. Parasit Vectors. 2018;11:596.
22. Mehrizi AA, Rezvani N, Zakeri S, Gholami A, Babaeekhou L. Poly(I:C) adjuvant strongly enhances parasite-inhibitory antibodies and Th1 response against Plasmodium falciparum merozoite surface protein-1 (42-kDa fragment) in BALB/c mice. Med Microbiol Immunol. 2018;207:151-66.

23. Perez-Leal O, Sierra AY, Barrero CA, Moncada C, Martinez P, Cortes J, et al. Plasmodium vivax merozoite surface protein 8 cloning, expression, and characterisation. Biochem Biophys Res Commun. 2004;324:1393-9.

24. Bull PC, Lowe BS, Kortok M, Molyneux CS, Newbold Cl, Marsh K. Parasite antigens on the infected red cell surface are targets for naturally acquired immunity to malaria. Nat Med. 1998;4:358-60.

25. Silva JC, Egan A, Friedman R, Munro JB, Carlton JM, Hughes AL. Genome sequences reveal divergence times of malaria parasite lineages. Parasitology. 2011;138:1737-49.

26. Rutledge GG, Böhme U, Sanders M, Reid AJ, Cotton JA, Maiga-Ascofare $\mathrm{O}$, et al. Plasmodium malariae and $P$ ovale genomes provide insights into malaria parasite evolution. Nature. 2017;542:101-4.

27. Flück C, Smith T, Beck HP, Irion A, Betuela I, Alpers MP, et al. Strain-specific humoral response to a polymorphic malaria vaccine. Infect Immun. 2004;72:6300-5.

28. Franks S, Baton L, Tetteh $K$, Tongren E, Dewin D, Akanmori BD, et al. Genetic diversity and antigenic polymorphism in Plasmodium falciparum: extensive serological cross-reactivity between allelic variants of merozoite surface protein 2. Infect Immun. 2003;71:3485-95.

29. Osier FH, Polley SD, Mwangi T, Lowe B, Conway DJ, Marsh K. Naturally acquired antibodies to polymorphic and conserved epitopes of Plasmodium falciparum merozoite surface protein 3. Parasite Immunol. 2007:29:387-94.

30. Blackman MJ, Heidrich HG, Donachie S, McBride JS, Holder AA. A single fragment of a malaria merozoite surface protein remains on the parasite during red cell invasion and is the target of invasion-inhibiting antibodies. J Exp Med. 1990:172:379-82.

31. Gowda DC. TLR-mediated cell signaling by malaria GPIs. Trends Parasitol. 2007;23:596-604.

32. Ferreira MU, Kimura EA, De Souza JM, Katzin AM. The isotype composition and avidity of naturally acquired anti-Plasmodium falciparum antibodies: differential patterns in clinically immune Africans and Amazonian patients. Am J Trop Med Hyg. 1996;55:315-23.
Ready to submit your research? Choose BMC and benefit from:

- fast, convenient online submission

- thorough peer review by experienced researchers in your field

- rapid publication on acceptance

- support for research data, including large and complex data types

- gold Open Access which fosters wider collaboration and increased citations

- maximum visibility for your research: over $100 \mathrm{M}$ website views per year

At BMC, research is always in progress.

Learn more biomedcentral.com/submissions 